\title{
Experimental Study on Large Gap Magnetic Fluid Rotary Sealing of High Power Motor
}

\author{
Feifei Xing ${ }^{1,}$, Jun $\mathrm{Ji}^{2, \mathrm{~b}}$ \\ ${ }^{1}$ BMEI CO.,LTD., Beijing 100027, China \\ ${ }^{2}$ Beijing Polytechnic, Beijing 100176,China

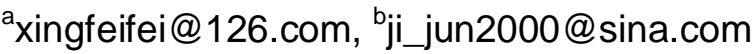

Key words: Magnetic fluid; Experiment; Sealing;Large gap

Abstract: The magnetic fluid large gap of rotary Sealing structure device of megawatt power motors have been designed, and the experimental of this structure was discussed. The results shows that, when the sealing gap were greater or equal to $0.3 \mathrm{~mm}$, the pressure capacity of this structure of high power motor can meet sealing requirements, and the pressure capacity was mainly related with the sealing stage, magnetic field strength, magnetic fluid saturation magnetization.

\section{Introduction}

Magnetic fluid is a colloidal suspension of subdomain-sized magnetic particles that exhibits very interesting properties under applied magnetic flied ${ }^{[1]}$. Magnetic fluid sealing is a new form of sealing method, it is rely on the liquid material to fill the gap to achieve sealing function. It has many advantages: zero leakage, long life,no pollution and high reliability. In many occasions, the gap of sealing structure is large, such as sealing megawatt power motors, it is necessary of the seal gap is more than or equal to $0.3 \mathrm{~mm}$, but It's typical sealing gap is $0.1 \mathrm{~mm}-0.3 \mathrm{~mm}^{[2]}$. In these situation, use the traditional sealing method may have some difficulties: poor sealing functions, difficult to process the components , difficult to assemble, complex structures and so on ${ }^{[3-4]}$.It is more necessary to study the large gap under the magnetic fluid sealing technology. This paper used the rotation sealing of ferrofluid for high power electric motor, the application context for the special requirements of the ferrofluid sealing have not been submitted in civil technology. It presents a magnetic fluid rotary sealing structure, the gap can be adjusted from $0.3 \mathrm{~mm}$ to $0.6 \mathrm{~mm}$, it was also tested the pressure capacity and the leakage rate under the corresponding sealing gap. It had been designed the large size large gap rotating sealing device for the characteristics of the high power electric motor, because it was difficult for large size motor assembly, it can also designed the split structure.

\section{The principle of magnetic fluid sealing}

The operation principle of a usual magnetic fluid shaft sealing is shown as (Fig.1 ${ }^{[5]}$. It consists of a permanent ring magnet, two pole pieces, magnetic fluid, and a magnetically permeable shaft, the magnetic fluid and the magnetically permeable shaft which is to be sealed. All these parts together form a closed magnetic circuit. The sharp-edged structures in the pole pieces (or in the shaft) locally build up a very small clearance between the dynamic (shaft) and static(pole piece). 


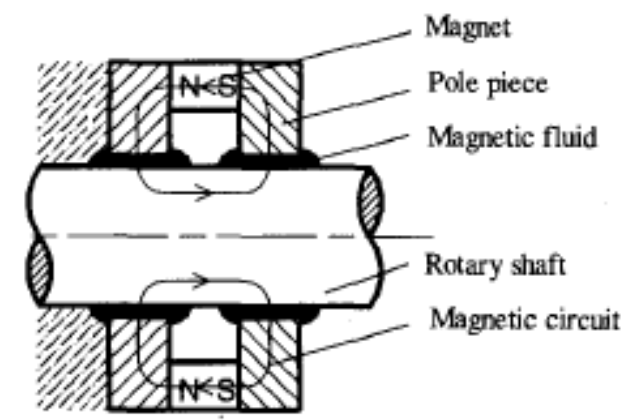

Fig.1 Principle diagram of typical magnetic fluid sealing structure

Usually, the magnetic fluid seal's pressure resistance of each single-stage is ${ }^{[6]}$

$\Delta p \approx n \mu_{0} M_{s} \nabla H \approx n \mu_{0} M_{s}\left(H_{\max }-H_{\min }\right)$

Where the $\mu_{0}$ is the vacuum permeability, $M_{s}$ is the saturation magnetization of magnetic fluid, $\mathrm{n}$ is the number of pole piece, $\mathrm{H}$ is the magnetic field intensity in the seal gap, $H_{\max }$ and $H_{\text {min }}$ is the maximum and the minimum of the magnetic field intensity within the magnetic fluid region $^{[6]}$.

\section{The experiments of magnetic fluid static sealing.}

The magnetic fluid large gap of rotary Sealing structure device of megawatt power motors was designed as figure 2 shows, it includes the sealing chamber, permanent magnet, the different shapes of magnetic pole pieces and so on. There are four permanent magnets, each of them with its corresponding permanent magnet pole pieces can constitute more magnetic circuits, the four teeth structures are the triangular shapes, the fist pole piece is rectangular,and the total umber of the tooth is 9 , each triangula teeth have the same angle.

The sealing chamber housing is $20 \#$ stainless steel materials, the permanent magnet material is $\mathrm{NdFeB}$, it can form high strength magnetic field. The pole pieces are the iron, and the shaft is 304 steel, we can get the different shaft sealing gaps by adjustment the center shaft.

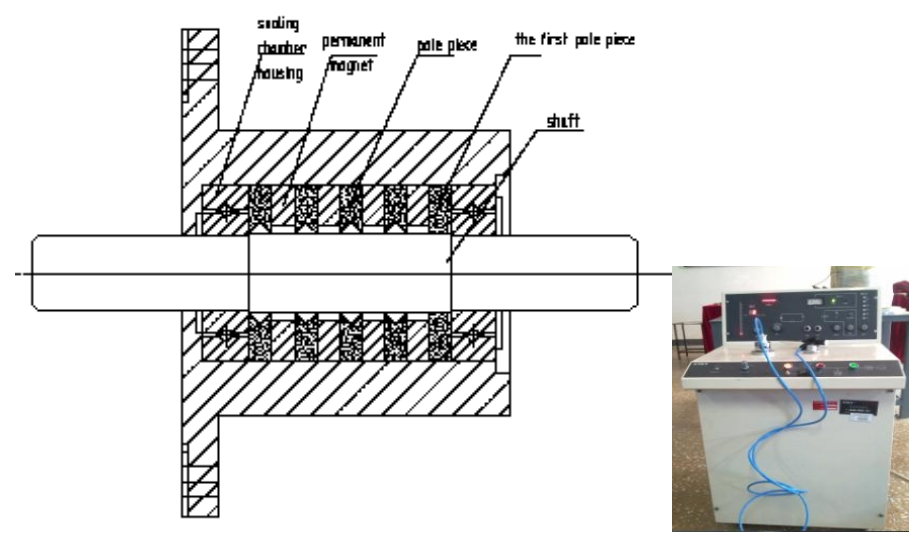

Fig.2 Device of magnetic fluid sealing and the helium leak detectors

For leak detection process, using suction leak marksmanship principles shown in the Figure 2. Sealed chamber dedicated sniffing leak detection port is connected to the instrument, connect the 
magnetic fluid seals are filled with a certain proportion of helium - nitrogen gas mixture. When the leak, so that the suction gun along the drain hole suspicious move slowly, if tainted hole, helium leak from the drain hole is sucked to the suction gun tube mass spectrometry instrument is detected.

The experiment was making the high-pressure helium continuously fill the sealing chamber by regulating the valve. In order to determine the different gaps, at different pressures, the magnetic fluid seal leak rate, measured in the laboratory bench using suction gap marksmanship are $0.3 \mathrm{~mm}$, $0.4 \mathrm{~mm}, 0.5 \mathrm{~mm}, 0.6 \mathrm{~mm}, 0.7 \mathrm{~mm}$ of pressure under different leakage rate, as shown in table 1 . The data show in Table 1.

\begin{tabular}{|c|c|c|c|c|c|}
\hline Sealing gaps $/ \mathrm{mm}$ & 0.3 & 0.4 & 0.5 & 0.6 & 0.7 \\
\hline Pressure capacities/MPa & 0.52 & 0.48 & 0.36 & 0.28 & 0.23 \\
\hline The pressure capacities after self-repairing/MPa & 0.32 & 0.29 & 0.17 & 0.12 & 0.10 \\
\hline
\end{tabular}

According to Table 1 of the data,we can see that :for a typical seal gap $0.15 \mathrm{~mm}^{[7-8]}$, single seal can withstand a pressure of about $21 \mathrm{KPa}$, and this article is designed sealing structure, single-stage sealing gap $0.4 \mathrm{~mm}$ can withstand the pressure has reached about $38 \mathrm{KPa}$. With the increase of the sealing gap, the pressure value decreases. The maximum gap $0.7 \mathrm{~mm}$ single-stage pressure capacity could meet $18 \mathrm{KPa}$. In addition, the magnetic fluid seal also has a large gap self-healing function, after the ester-based magnetic liquid is broken high-voltage charge, but also to maintain a certain pressure capacity.

\section{The Experiments of Large gap magnetic fluid seal self-healing function}

In order to fully illustrate ester-based magnetic fluid in the large gap under the self-healing effect of the sealing function, we take the gap of $0.6 \mathrm{~mm}$ and $0.7 \mathrm{~mm}$ pressurized repeated experiments, when the magnetic liquid seal-breaking maximum charge pressure value (destruction), the stop inflatable, this time gauge will slowly drop to a certain value has stabilized; after a period of time to continue to the sealed chamber pneumatic compression, a process is repeated three times before; test the final steady pressure. The data obtained are shown in Table 2 (a) (b).

Tab 2(a) Magnetic fluid pressure capability of $0.6 \mathrm{~mm}$ gap

\begin{tabular}{ccccc}
\hline Stamping number & $\begin{array}{c}\text { The first } \\
\text { time }\end{array}$ & The second time & the third time & the fourth time \\
\hline $\begin{array}{c}\text { The maximum pressure } \\
\text { value (MPa) } \\
\text { Repair pressure value } \\
(\mathrm{MPa})\end{array}$ & 0.23 & 0.18 & 0.1 & 0.04 \\
\hline
\end{tabular}


Tab 2(b) Magnetic fluid pressure capability of $0.7 \mathrm{~mm}$ gap

\begin{tabular}{ccccc}
\hline Stamping number & $\begin{array}{c}\text { The first } \\
\text { time }\end{array}$ & The second time & the third time & the fourth time \\
\hline $\begin{array}{c}\text { The maximum pressure } \\
\text { value (MPa) } \\
\text { Repair pressure value } \\
(\mathrm{MPa})\end{array}$ & 0.22 & 0.17 & 0.14 & 0.10 \\
\hline
\end{tabular}

\section{Summary}

(1) The magnetic fluid large gap of rotary Sealing structure device of megawatt power motors have been, when the sealing gap were greater or equal to $0.3 \mathrm{~mm}$, the pressure capacity of this structure of high power motor can meet sealing requirements.

(2) The magnetic fluid seal also has a large gap self-healing function, after the ester-based magnetic liquid is broken high-voltage charge, but also to maintain a certain pressure capacity.

(3) In the present experimental conditions, the magnetic fluid "zero" leakage conclusion is still valid.

\section{References}

[1] Liu T G,Liu S J, Yang Z Y. Preparation of magnetic fluids used for sealing lubricating oil. Journal of China University of Mining and Technology,2004,33(5):543-545.

[2]B.Ando, A.Ascia, S.Baglio et al.Magnetic fluids and their use in transducers[J]. IEEE Instrumentation and measurement magazine.2006.9(6):44-47

[3]R.E.Rosensweig. Heating magnetic fluid with alternating magnetic field[J]. Journal of Magnetism and magnetic materials. 2002.252:370-374

[4] D.C.Li, H.P. Xu, X.Z. He et al. Mechanism of magnetic liquid flowing in the magnetic liquid seal gap of reciprocating shaft[J]. Journal of Magnetism and Magnetic Materials.2005.289:407-410

[5] Z.X.Li. Magnetic fluid seals for DWDM filter manufacturing[J]. Journal of Magnetism and Magnetic Materials. 2002.252:327-329

[6]D.C.Li, H.P. Xu, X.Z. He,et al. Study on the magnetic fluid sealing for dry Roots pump[J]. Journal of Magnetism and Magnetic Materials. 2005.289:419-422.

[7]Feifei Xing, Decai Li, Xiaolong Yang. Experimental Study of Large Gap Magnetic Fluid Static Sealing. Key Engineering Materials.492(2012):283-286.

[8]G. Morton, W.G. Fiuh. The force on an object passing through a magnetic fluid seal[J]. Journal of Magneticsm and Magnetic Materials.2002.252:324-326 